\title{
analychealistry
feature
}

\section{Bipolar Electrodes: A Useful Tool for \\ Concentration, Separation, and Detection of Analytes in Microelectrochemical Systems}

\author{
François Mavré \\ Université Paris Diderot (France)

\begin{abstract}
Robbyn K. Anand, Derek R. Laws, Kwok-Fan Chow, Byoung-Yong Chang, John A. Crooks, and Richard M. Crooks
\end{abstract}

The University of Texas at Austin

Over the past decade, bipolar electrochemistry has emerged from relative obscurity to provide a promising new means for integrating electrochemistry into lab-ona-chip systems. This article describes the fundamental operating principles of bipolar electrodes, as well as several interesting applications.

A bipolar electrode (BPE) is an electronic conductor in contact with an ionically conductive phase. When a sufficiently high electric field is applied across the ionic phase, faradaic reactions occur at the ends of the BPE even though there is no direct electrical connection between it and an external power supply. In this article, we describe the fundamental principles and some electroanalytical applications of BPEs for array-based sensing, separations, and concentration enrichment in microelectrochemical systems. Specifically, we show how the latter three operations, which are normally thought of as arising from different phenomena, are linked by processes occurring on and near BPEs confined within a convenient, miniaturized microfluidic format. The results presented here demonstrate that under a particular set of conditions, up to 1000 well-defined BPEs can be simultaneously activated and interrogated using just a single pair of driving electrodes. Furthermore, a slight change to the resistance of the buffer solution within the microfluidic channel leads to the separation and concentration enrichment of charged analytes.

\section{OVERVIEW OF BIPOLAR ELECTROCHEMISTRY}

A traditional three-electrode electrochemical cell, which consists of a working electrode, an auxiliary electrode, and a

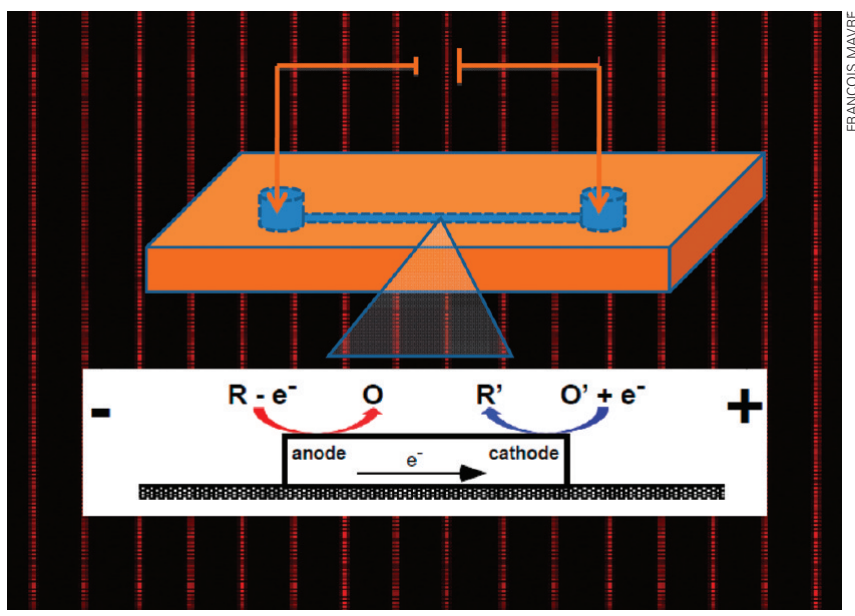

reference electrode, is illustrated in Scheme 1a. In this configuration, the potential of the working electrode, which is related to the energy of the electrons in the electrode, is controlled (versus a reference electrode) using a potentiostat. The potential of the solution is not directly controlled; in other words, it is at a floating potential that (in the absence of an externally applied electric field) depends on the composition of the solution. When the potential of the working electrode is set to a value more negative than that of an electroactive molecule in the solution, electrons may (depending upon kinetics) transfer from the electrode to reduce species in solution (Scheme 1b; note that positive potentials are up in this diagram to make it consistent with Scheme 1c). Similarly, oxidation reactions occur when the electron transfer is in the opposite direction. The faradaic current measured in the circuit connecting the working and auxiliary electrodes is a direct 
a

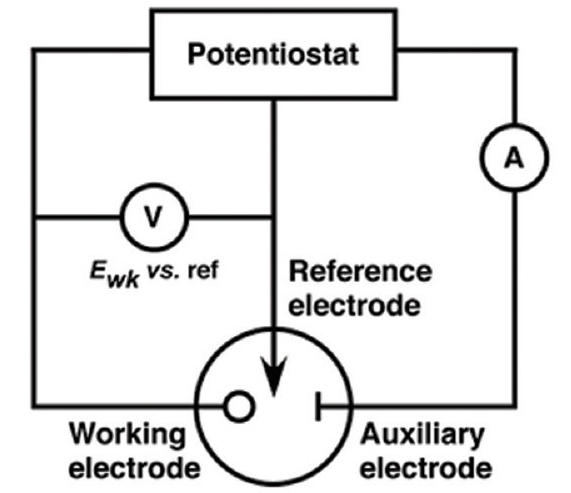

b

Potential

${ }^{+}$

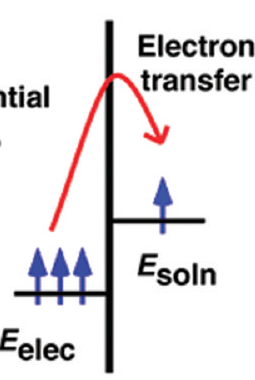

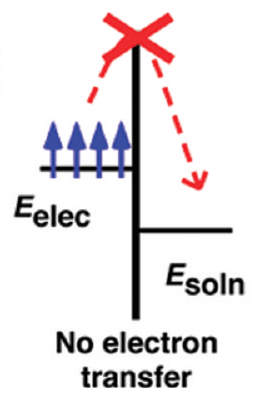

No electron
transfer

measure of the rate of this electrochemical reaction. The key point is that the interfacial potential difference between the electrode and the solution, rather than the absolute potential of either, is the driving force for electron transfer. ${ }^{1}$

A BPE has a configuration somewhat opposite to that of a conventional system; that is, the potential difference between the electrode and the solution is determined by the electric field in solution. Despite the reversed roles of the solution and electrode in a BPE experiment, control is still maintained over the interfacial potential difference. The experimental configuration of BPEs is illustrated in Scheme 1c. A conductive material, which has no external electrical connection, is located inside a microchannel whose dimensions are generally small enough to ensure a high resistance to current flow. For example, in most of our experiments the channel is $1 \mathrm{~cm}$ long, $20 \mu \mathrm{m}$ high, and $1 \mathrm{~mm}$ wide. The BPE is typically $0.5-1.0 \mathrm{~mm}$ in length. A simple power supply applies a potential difference, $E_{t o t}$, between two driving electrodes situated in reservoirs at both ends of the channel. Because the channel resistance is high, most of $E_{t o t}$ is dropped linearly along the channel length. Depending upon the experimental conditions, $\sim 10-15 \%$ of $E_{\text {tot }}$ is lost at the driving electrode/solution interface (Scheme 1c). ${ }^{2}$

Comparison with an analogous electrical circuit comprised of parallel resistors (Scheme 2a and 2b) can help illustrate how a $\mathrm{BPE}$ responds to $E_{t o t}$ and how it interacts with the electrolyte solution in the microchannel. The total current in the channel $\left(i_{\text {channel }}\right)$ can be carried either through the solution by ion migration $\left(i_{s}\right)$ or through the electrode via electrons generated by faradaic processes $\left(i_{b p e}\right)$ at the BPE (note that we neglect the capacitance of the BPE in this treatment). The relative fraction of the current passing through the solution and the electrode depends on the relative values of $R_{s}$, which represents the resistance of the electrolyte solution, and $R_{e}$, which is the

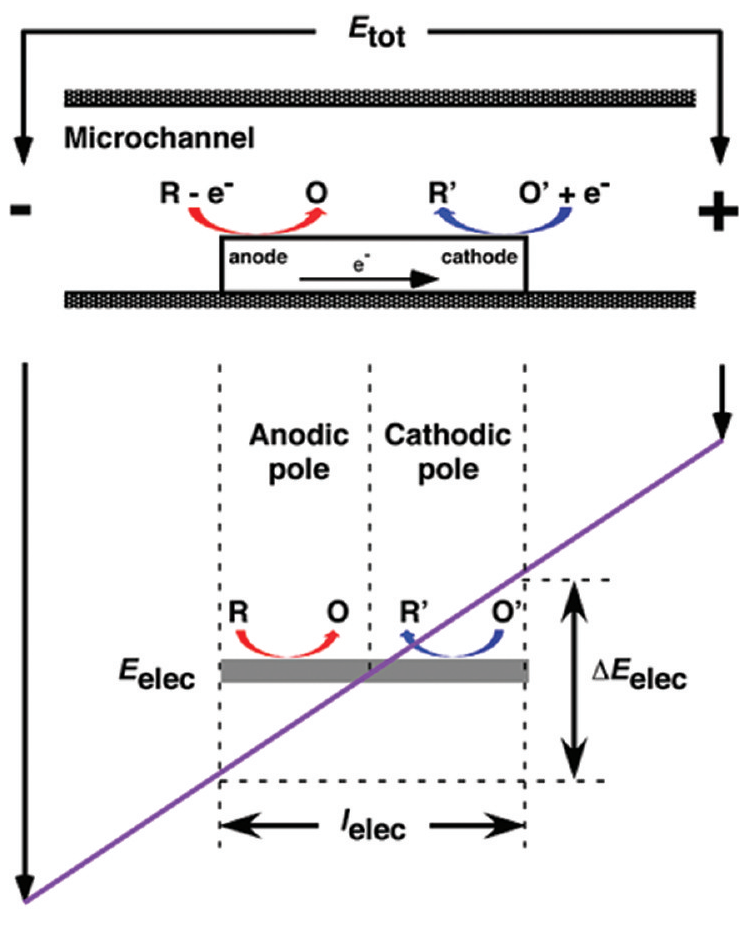

Scheme 2

a
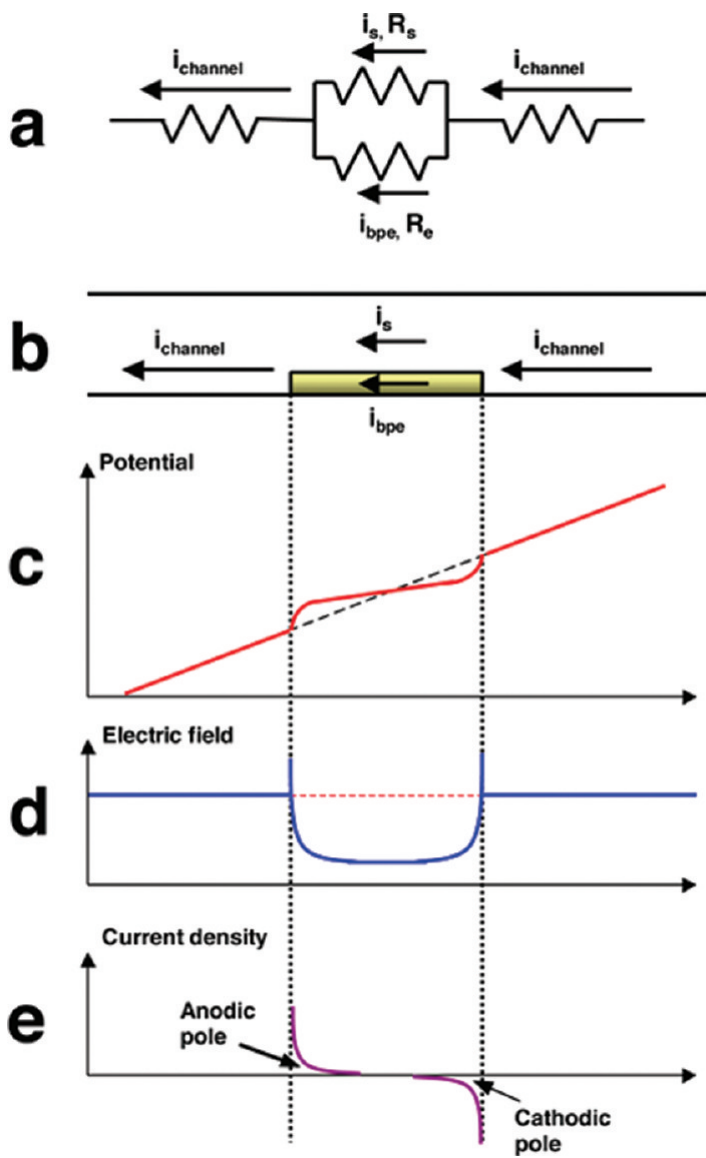

global resistance of the BPE to faradaic electrochemistry arising from both electron and mass transfer effects, as given in Equation 1. 


$$
\frac{i_{\text {bpe }}}{i_{\text {channel }}}=1-\frac{i_{s}}{i_{\text {channel }}}=\frac{R_{s}}{R_{e}+R_{s}}
$$

The potential difference between the solution and the two ends of the BPE ( $\Delta E_{\text {elec }}$, Scheme 1c), which is roughly a function of $E_{t o t}$ and the length of the electrode $\left(l_{\text {elec }}\right)$, strongly influences $R_{e}$ : if the current is kinetically controlled, then a higher value of $\Delta E_{\text {elec }}$ results in a lower $R_{e}$. Likewise, Equation 1 predicts that higher $R_{s}$ results in more current passing through the BPE. At the extreme, as $R_{s}$ becomes very high, most of the channel current $\left(i_{\text {channel }}\right)$ passes through the BPE.

Faradaic Reactions at Bipolar Electrodes. The bipolar effect can be understood more completely if one considers the variation of the potential difference at the electrode/solution interface along the entire length of the electrode. Scheme $1 \mathrm{c}$ shows that a lateral potential drop in the electrolyte solution results in the potential of the electrode floating to an equilibrium value, $E_{\text {elec }}$, that separates the BPE into two poles: the cathodic pole, where the solution potential is higher than $E_{\text {elec }}$, and the anodic pole, where the solution potential is lower than $E_{\text {elec }}$. Accordingly, there is a potential difference at each lateral position of the electrode/solution interface that may or may not be sufficient to drive an electrochemical reaction. These cathodic and anodic electrochemical processes are coupled electrically via the electrode, and they must occur simultaneously and at the same rate to ensure that electroneutrality is maintained within the conductive BPE. This relationship means that the total current flowing through each pole of the BPE is the integrated sum of the current at every location in that pole (Scheme 2e).

Excluding mass transfer effects, the current density at the BPE mainly depends on two parameters: the overpotential available at every location at the electrode/solution interface and the kinetic characteristics of the redox couples involved in the faradaic processes. As mentioned earlier, $\Delta E_{\text {elec }}$ is the fraction of $E_{t o t}$ that is dropped across the BPE (Scheme 1c), and it represents the total driving force available to couple the two faradaic reactions at the poles of the BPE. For these two redox processes to occur simultaneously, the value of $\Delta E_{\text {elec }}$ has to be higher than the difference in the formal potentials for the two reactions. ${ }^{2}$ The $\Delta E_{\text {elec }}$ available in the system can be approximated by Equation 2 when $R_{s} \ll R_{e}$ (that is, when most of the current flowing in the microchannel is ionic).

$$
\Delta E_{\text {elec }}=\frac{E_{\text {tot }}}{l_{\text {channel }}} l_{\text {elec }}
$$

It follows that for a given electric field $\left(\sim E_{\text {tot }} / l_{\text {channel }}\right)$, longer electrodes yield a higher value of $\Delta E_{\text {elec }}$, resulting in a larger value of $i_{b p e}$. Consequently, the value of $E_{\text {tot }}$ required to induce faradaic reactions varies depending both on the species present in solution and on the ratio of electrode length to channel length. For most of the results reported by our group, $E_{\text {tot }}$ is in the range of $20-30 \mathrm{~V}$ and never $>100 \mathrm{~V}$. Therefore, inexpensive power supplies are sufficient to carry out many different kinds of interesting experiments with BPEs.

Modulation of the Local Electric Field in the Channel. When a portion of $i_{\text {channel }}$ is carried through the BPE, the potential drop above the electrode might deviate from linearity (Scheme 2c). That is, faradaic current results in a local decrease of the electric field above the electrode (Scheme 2d) caused by a reduction in $i_{s}$. Duval and coworkers call this phenomenon "faradaic depolarization".,4

When the BPE and the channel have the same width, Duval has shown that faradaic reactions alter the electric field over the electrode according to Equation $3:^{3}$

$$
\frac{\partial V(x, t)}{\partial x}=\frac{j(x, t)}{h K_{S}}
$$

Here, $V(x, t)$ is the electric field strength, $j(x, t)$ is the current density, $h$ is the height of the channel, and $K_{S}$ is the conductivity of the solution.

According to Equation 3, the variation of the electric field is directly proportional to the current density and depends on two additional parameters: the height of the channel and the conductivity of the solution. The extent of depolarization may be easily probed through the $i_{b p e} / i_{\text {channel }}$ ratio. Clearly, smaller channel heights and lower solution conductivities (e.g., lower electrolyte concentration) favor a higher degree of faradaic depolarization. Consequently, applications involving concentration enrichment, which are discussed at the end of the next section and which rely on faradaic depolarization, typically employ small channel heights and low electrolyte concentrations.

\section{APPLICATIONS OF BIPOLAR ELECTRODES}

Background. Although BPEs have not been widely adopted for electroanalytical purposes, they have been used for many years in battery technologies, for electrosynthesis, ${ }^{5,6}$ as photoelectrodes for solar cells, ${ }^{7-9}$ and as seeds to generate nanowires along electric field lines. ${ }^{10}$ More recently, Sen, Mallouk, and coworkers have shown that bipolar electrochemistry can lead to selfpropulsion of freely suspended nanorods. ${ }^{11}$ In this experiment, a bimetallic $\mathrm{Pt} / \mathrm{Au}$ nanorod electrocatalyzes the oxidation and reduction of $\mathrm{H}_{2} \mathrm{O}_{2}$ at the $\mathrm{Pt}$ and $\mathrm{Au}$ sites, respectively, and this leads to predictable motion of the nanorods. Bipolar electrochemistry has also been used to prepare surfaces modified with gradients of various materials. This application relies on the axial gradient in the potential difference between the solution and BPE discussed earlier (Scheme 1c). Using this principle, molecular gradients of self-assembled monolayers have been prepared on Au surfaces, ${ }^{12,13} \mathrm{Au}$ has been electrodeposited onto the ends of carbon nanotubes, ${ }^{14}$ and graded compositions of $\mathrm{CdS}$ have been synthesized. ${ }^{15}$

The remainder of this article focuses on very recent electroanalytical applications of BPEs with an emphasis on results from our own laboratory and that of our collaborators Prof. Ulrich Tallarek and Dr. Dzmitry Hlushkou of Philipps-Universität Marburg. As alluded to earlier, the main advantage of BPEs for electroanalysis is the ease of controlling their potential: a simple power supply, or even a battery, suffices, and no direct electrical contact is required. However, the latter point makes it difficult to measure current flowing through the electrode. In the following sections, we describe three approaches that have been used to overcome this problem (Scheme 3).

Direct Detection of Current Using BPEs. BPEs can be particularly useful for detecting electroactive analytes in microfluidic environments, where high electric fields and solution 
Scheme 3
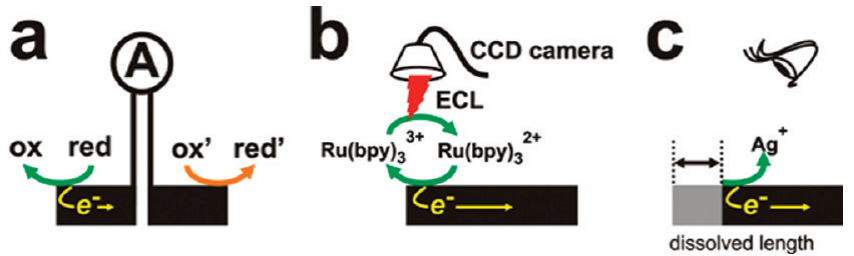

resistances can lead to difficulties controlling the potential of a working electrode configured in a standard three-electrode cell arrangement. ${ }^{16}$ For example, Nyholm and coworkers have used BPEs to detect electroactive molecules in a capillary ${ }^{17}$ and in an on-chip microfluidic device ${ }^{18}$ by taking advantage of the electric field used for electrophoresis to induce bipolar behavior between two Au microbands that were connected externally through an ammeter (Scheme 3a). This kind of split electrode design makes it possible to directly measure the current passing through the $\mathrm{BPE}$, but at the cost of complicating the system with an external electrical connection.

Detection of Electroactive Molecules Using Electrogenerated Chemiluminescence (ECL) Reporting. An alternative strategy for detecting faradaic processes at BPEs is to use ECL as an indirect reporter of the current (Scheme 3b). ${ }^{19,20}$ This approach eliminates the need for a direct external connection to the electrode and opens up the possibility of using optical detection to simultaneously detect faradaic current at many BPEs. Because ECL does not require an excitation light source, it is generally superior to fluorescence for low-cost and portable sensor systems. $^{21}$

One of the best known ECL systems uses Ru(bpy) ${ }_{3}{ }^{2+}$ as the light-emitting species and an amine, such as tri- $n$-propylamine (TPrA), as a co-reactant. ${ }^{21}$ Using this approach, Manz and coworkers demonstrated ECL generation at the anodic pole of a Pt BPE. ${ }^{19}$ Specifically, they showed that the reaction of $\mathrm{Ru}(\mathrm{bpy})_{3}{ }^{2+}$ with different amines or amino acids provides a means for signaling the presence of analytes electrophoretically separated in a microfluidic system. However, this detection scheme was limited to just the detection of ECL co-reactants, although other analytes could be detected if they interfered with the ECL reaction and thereby reduced light emission from the BPE.

The Manz approach to ECL-based detection only took advantage of the anodic pole of the BPE, and therefore it was limited to detection of analytes that specifically interacted with the ECL process. Shortly after their report, however, we demonstrated that the presence of any electroactive analyte could be detected by taking advantage of both poles (Scheme $3 b$ ). Here, the analyte of interest is reduced at the cathodic pole, and this faradaic reaction triggers light emission at the anodic pole by the corresponding oxidation of $\mathrm{Ru}(\mathrm{bpy})_{3}{ }^{2+}$ and $\mathrm{TPrA}^{20}$ This finding opened the way for detection of a much larger variety of compounds, ${ }^{22,23}$ including large biomolecules such as DNA. ${ }^{24}$

The BPE experiment illustrated in Scheme $3 \mathrm{~b}$ is usually carried out in a microfluidic device, which provides a means for controlling the electric field in the microchannel and providing for flow of analyte solutions. Microfluidic devices used in our lab are usually comprised of a PDMS block that is irreversibly attached to a glass slide onto which one or more Au BPEs have been microfabricated (Scheme
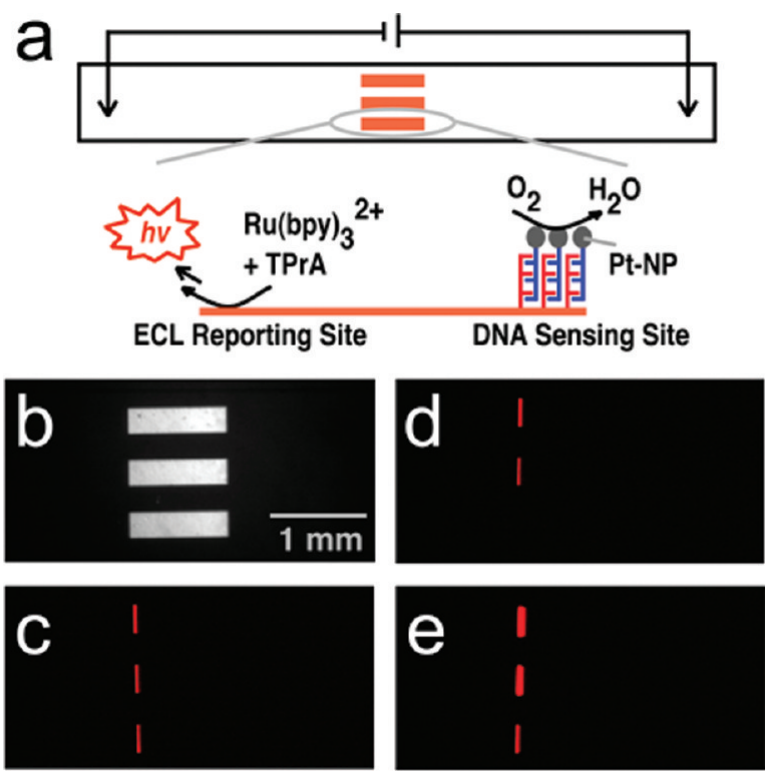

Figure 1. (a) A top-view schematic of a microdevice showing three BPEs and the driving electrodes and an illustration showing an approach for sensing DNA. (b) Optical micrograph showing the BPE array in the microchannel. False-color luminescence micrographs showing: (c) ECL emitted at $E_{\text {tot }}=16.0 \mathrm{~V}$ when complementary target DNA functionalized with Pt-NPs is hybridized to probe DNA present on the electrode surface, (d) the ECL emitted at $16.0 \mathrm{~V}$ when only the top two electrodes of the device are exposed to the labeled target, and (e) the ECL emission at $E_{\text {tot }}=22.0 \mathrm{~V}$ for the device in (d).

1c). ${ }^{2}$ The Au electrodes, situated in the middle of the channel, are typically $0.5-1.0 \mathrm{~mm}$ long. At the two ends of the channel, macroscopic reservoirs allow for channel filling and serve as the locations of the driving electrodes used to apply $E_{\text {tot }}$. For ECL reporting experiments, the solution filling the channel contains $\mathrm{Ru}(\mathrm{bpy})_{3}{ }^{2+}$, TPrA, an electrolyte or buffer, and the analyte of interest. Upon application of a sufficiently high value of $E_{t o t}$, $\mathrm{Ru}(\mathrm{bpy})_{3}{ }^{2+}$ and TPrA are both oxidized at the anode and the analyte is reduced at the cathode (Scheme $3 b$ ). Several years ago, we demonstrated the principle of this double-pole ECL reporting scheme by detecting benzyl viologen $\left(\mathrm{BV}^{2+}\right)$ at an indium tin oxide (ITO) $\mathrm{BPE}^{20}$ The relationship between $\mathrm{BV}^{2+}$ concentration in the channel and ECL intensity was shown to increase linearly until complications arising from quenching occurred. Later, using a split BPE, we demonstrated a linear correspondence between the ECL emission intensity and the rate of the cathode reaction. ${ }^{2}$ Finally, measured ECL intensity and bipolar current were found to be in good agreement with calculated values based on the kinetics of the electrochemical processes. ${ }^{2}$

Microelectrochemical Arrays Based on BPEs Coupled to ECL Emission. BPEs provide a means to vastly simplify the design of microelectrochemical arrays. ${ }^{24,25}$ For example, Figure 1a shows the basic design of a small, wireless DNA microelectrochemical array. It consists of three $\mathrm{Au}$ BPEs $(1.00 \times 0.25 \mathrm{~mm})$ housed at the center of a PDMS microchannel $(1.20 \mathrm{~cm}$ long, $1.75 \mathrm{~mm}$ wide, and $28 \mu \mathrm{m}$ high) filled with an aqueous solution of $\mathrm{Ru}(\mathrm{bpy})_{3}{ }^{2+}$ and TPrA (Figure 1b). The cathodic poles of all three BPEs are modified with the same sequence of probe DNA. The driving voltage in this experiment is set to $16.0 \mathrm{~V}\left(\Delta E_{\text {elec }}=1.33 \mathrm{~V}\right)$, which is sufficient to initiate ECL emission at the anodic pole and $\mathrm{O}_{2}$ reduction at the cathodic pole (Figure 1a), but only in the presence of a Pt catalyst. 

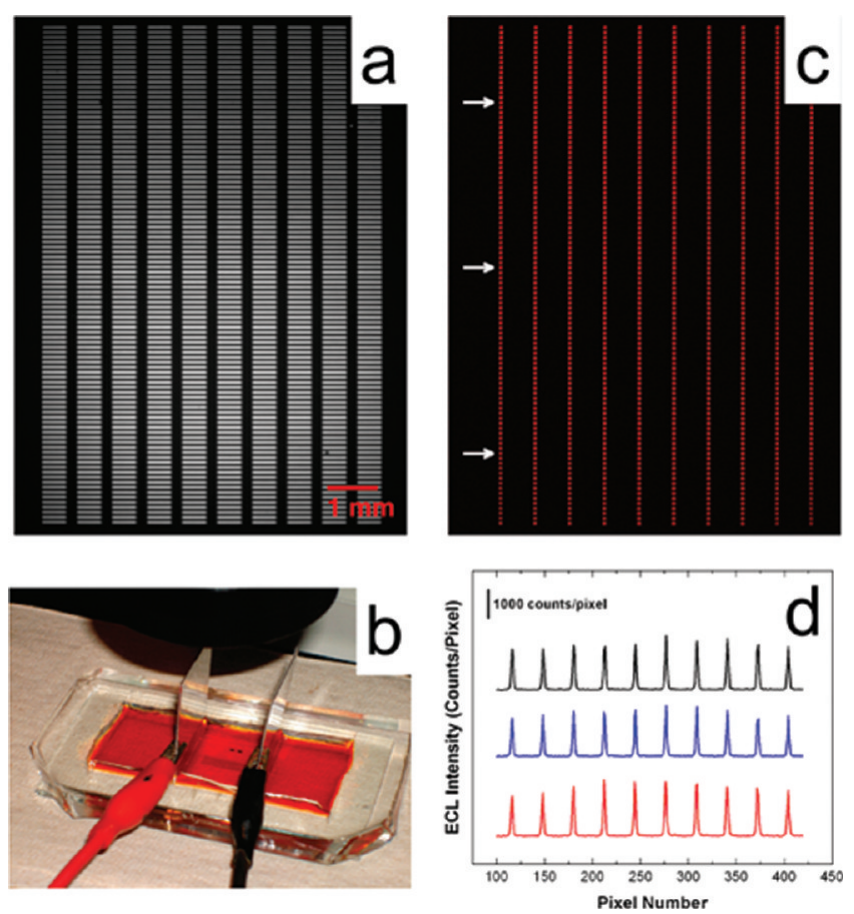

Figure 2. (a) Optical micrograph of an array comprising 1000 individual BPEs having dimensions of $500 \times 50 \mu \mathrm{m}$. (b) Photograph of the cell configuration used to activate this array. (c) Luminescence micrograph showing the ECL response of the array when $E_{\text {tot }}=85.0$ V. (d) ECL intensity profile obtained along the rows of BPEs indicated by arrows in (c).

DNA is detected by exposing the array to a complementary target bearing a 4-nm Pt nanoparticle label. Upon hybridization, the label catalyzes $\mathrm{O}_{2}$ reduction; the electrons required for this reaction originate from oxidation of $\mathrm{Ru}(\mathrm{bpy})_{3}{ }^{2+}$ and TrPA. Hence, ECL is emitted from the anodic poles of the BPEs (Figure 1c). When the same experiment is carried out with only the top two electrodes exposed to the labeled target, the bottom electrode remains dark (Figure 1d). However, when $E_{\text {tot }}$ is increased to $22.0 \mathrm{~V}\left(\Delta E_{\text {elec }}=1.83 \mathrm{~V}\right), \mathrm{O}_{2}$ can be reduced directly on the $\mathrm{Au} \mathrm{BPE}$, and therefore ECL is now observed on the bottom electrode (Figure 1e). Note, however, that under these conditions, the emission intensity is still higher on the upper pair of electrodes, indicating that raising $E_{t o t}$ increases the rate of $\mathrm{O}_{2}$ reduction when the label is present.

The BPE approach is inherently scalable, and there should be no barrier to greatly increasing the number of electrodes in a microelectrochemical array while still retaining all the advantages of the just-described three-electrode array. Accordingly, we developed a microelectrochemical array consisting of $1000 \mathrm{Au}$ BPEs (Figure 2a). Each electrode is $500 \mu \mathrm{m}$ long and $50 \mu \mathrm{m}$ wide, and as before, they are all activated with a single pair of driving electrodes. However, the cell used to house this array was simplified compared to the microfluidic device described earlier. In the present case, the glass slide onto which the BPEs were microfabricated is simply dropped into a shallow pool containing electrolyte, $\mathrm{Ru}(\mathrm{bpy})_{3}{ }^{2+}$, and TPrA, and the driving electrodes are a pair of parallel plates that ensure a uniform electric field across the array (Figure $2 b$ ).

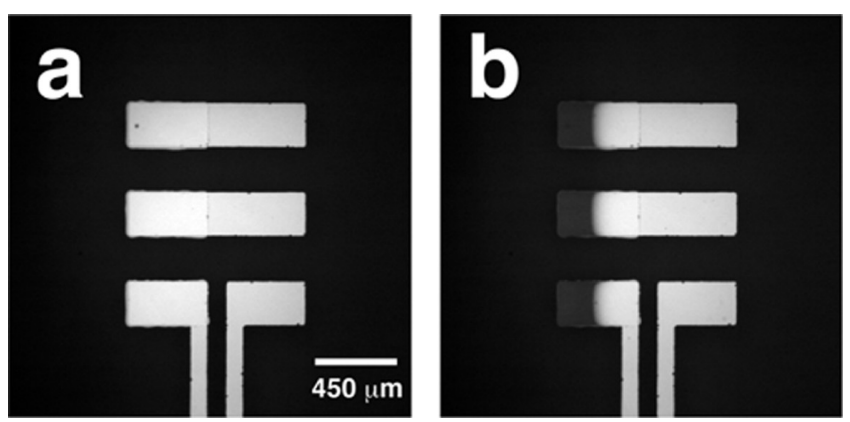

Figure 3. Optical micrographs showing an array of Ag-coated BPEs (including one split BPE) in the microchannel (a) before and (b) after application of $E_{\text {tot }}$ for $290 \mathrm{~s}$. The dark regions of the electrodes in (b) correspond to dissolution of $\mathrm{Ag}$. The solution contained $1.00 \mathrm{mM}$ p-benzoquinone in $0.10 \mathrm{M}$ acetate buffer at $\mathrm{pH} 5.5$.

When a sufficiently high value of $E_{t o t}$ is applied, the anodic poles of all 1000 electrodes emit ECL (Figure 2c). In this case, however, no DNA is involved. Rather, for demonstration purposes, the cathode reaction is simply reduction of $\mathrm{O}_{2}$ and $\mathrm{H}_{2} \mathrm{O}$ directly on the Au BPE. Figure $2 \mathrm{~d}$ shows emission intensity line profiles taken across the three rows of electrodes indicated with white arrows in Figure 2c. The uniform emission intensity demonstrates the viability of these large arrays for future sensing applications.

Bipolar Arrays Using Electrodissolution as a Sensing Modality. The ECL reporting method is highly effective for largescale BPE arrays, but it suffers from two shortcomings: first, it requires a sensitive optical detection system and second, the efficiency of the ECL reaction is rather low. ${ }^{2}$ Accordingly, we have developed a new read-out method that resolves both of these issues.

As shown in Scheme 3c, this method relies on electrodissolution of the BPE itself. Specifically, a layer of $\mathrm{Ag}$ metal is deposited onto the anodic pole of the BPE, and when a cathodic sensing event occurs, the Ag layer begins to dissolve. The extent of Ag electrodissolution provides a permanent record of the state of the BPE sensor. That is, because of charge neutrality, the amount of oxidized $\mathrm{Ag}$ must correspond to the number of electrons transferred at the cathodic pole of the BPE.

Figure 3 presents an experiment that illustrates this new sensing concept. The anodic poles of the three Au BPEs were coated with $5 \mathrm{~nm}$ of $\mathrm{Cr}$ and $20 \mathrm{~nm}$ of Ag. ${ }^{26}$ When the microchannel is filled with $1.0 \mathrm{mM}$ p-benzoquinone in acetate buffer at $\mathrm{pH} 5.5$ and $12.0 \mathrm{~V}$ is applied to the driving electrodes, the Ag layer starts to dissolve from the distal end of the BPE anode toward its center. The degree of shortening of the BPE is directly related to the amount of charge passed though the BPE. The latter point was verified by connecting an ammeter between the two poles of the split BPE at the bottom of the array. We recently demonstrated that this same general approach can be used to construct enzymebased biosensors for detecting DNA. ${ }^{26}$

Concentration Enrichment of Charged Analytes. Together with our collaborators, Prof. Ulrich Tallarek and Dr. Dzmitry Hlushkou of Philipps-Universität Marburg (Germany), we have recently been exploring a new use of BPEs: concentration enrichment and separation of analytes. ${ }^{27-30}$ As mentioned, this application takes advantage of the ability of a BPE to change the local electric field within a microfluidic channel.

Concentration enrichment, or preconcentration, is often required prior to separation and detection in techniques such as 
capillary or microchip electrophoresis. The majority of online concentration enrichment methods in electrophoresis employ a discontinuity in the buffer concentration or composition to induce an electric field gradient. This imparts a change in the migration velocity of charged analytes and can lead to sample stacking. ${ }^{31-34}$ Such methods can result in enrichment factors (EFs) of $100-1000 .^{34}$ However, all of these methods require preparation and precise injection of different buffers and solutions. Furthermore, they do not provide a practical means for both concentration enrichment and separation. Other preconcentration methods rely on the acid-base chemistry of the analyte, but in this case, resolution is limited by the steepness of the $\mathrm{pH}$ gradient. In a report that is more closely related to our recent findings, Yeung and coworkers used a BPE confined to a capillary to generate a $\mathrm{pH}$ gradient suitable for isoelectric focusing. ${ }^{35}$

The method of concentration enrichment we have been exploring takes advantage of the extended electric field gradient formed at the edge of a BPE in a low ionic strength buffer. Although still in the early days of development, this method has some desirable characteristics: it is exceedingly simple to implement (indeed, we discovered it by accident) and can not only enrich the concentration of the analyte, but also separate and detect solutions containing multiple analytes.

With one key exception, the experimental design required for this experiment is essentially identical to that used for the sensing experiments described earlier. The difference is that the concentration of the buffer must be lower for concentration enrichment: in the sensing experiments, it was typically $0.1 \mathrm{M}$, but here it is usually $1-5 \mathrm{mM}$. The higher resistance of the buffer solution shunts more current through the BPE (Scheme 2a), and this in turn leads to a greater degree of faradaic depolarization and hence the necessary distortion of the local electric field within the microchannel. The results presented here were obtained using microchannels with lengths, widths, and heights of $6 \mathrm{~mm} \times 100$ $\mu \mathrm{m} \times 21 \mu \mathrm{m}$, respectively, and 1 or $5 \mathrm{mM}$ Tris buffer. ${ }^{27-30}$

The phenomenon of electrokinetic concentration enrichment at a BPE arises from a redistribution of buffer ions in response to faradaic depolarization of the electrode. ${ }^{27,28}$ When a potential is applied across a microchannel, a cathodic electroosmotic flow (EOF) develops in the microchannel (right to left in Figure 4a). The electroosmotic mobility is $\sim 7.3 \pm 0.2 \times 10^{-4} \mathrm{~cm}^{2} \mathrm{~V}^{-1} \mathrm{~s}^{-1}$, which translates to a linear velocity of $4.3 \pm 0.1 \times 10^{-2} \mathrm{~cm} \mathrm{~s}^{-1}$ when $E_{t o t}=35 \mathrm{~V}$. Of utmost importance is the region of TrisH ${ }^{+}$ depletion near the cathodic edge of the BPE. Here, TrisH ${ }^{+}$is neutralized by reaction with $\mathrm{OH}^{-}$produced at the BPE cathode. This results in an ion depletion zone and hence a gradient in ionic strength, which is directly responsible for the formation of an extended electric field gradient in the anodic segment of the microchannel. We have recently confirmed the presence of the electric field gradient predicted by the simulations using appropriate experiments. ${ }^{30}$ Specifically, the type of continuous BPE illustrated in Scheme 1c was replaced with an array of 15 microband electrodes ( $40 \mu \mathrm{m}$ lines with $40 \mu \mathrm{m}$ spaces), which was also situated at the bottom of the microchannel, as illustrated in Figure $4 \mathrm{~b}$. When two of these microband electrodes are connected external to the microchannel by a conductive wire, they function as a BPE. The outer edge-to-edge distance of the two microbands determines the length of the BPE. The connected- microband BPE drives concentration enrichment, and the potential difference between neighboring pairs of the remaining microbands yields a map of the electric field profile (Figure 4c).

An anionic fluorescent tracer having a sufficiently low electrophoretic (EP) mobility, such as BODIPY disulfonate (BODIPY $492 / 515$, BODIPY ${ }^{2-}, \mu_{\mathrm{ep}}=-4.4 \times 10^{-4} \mathrm{~cm}^{2} \mathrm{~V}^{-1} \mathrm{~s}^{-1}$ ), can enter the microchannel by way of the cathodic EOF. As the analyte moves through the channel, an increasing electric field strength is encountered (Figure 4c). The velocity of the EP migration of BODIPY $^{2-}$ towards the anode increases until the electroosmotic and $\mathrm{EP}$ velocities just balance. This results in the formation of a concentrated band of dye (Figure 4a). The EF achieved increases with time and is dependent upon the initial concentration of BODIPY2- (Figure 4d). The steepness of the field gradient and the absolute field strength determine the intensity of the sequestering forces (EOF and electrophoresis) and in turn, the extent of enrichment.

Separation of Charged Analytes. According to the model discussed in the previous section, the location of concentration enrichment is dependent on the EP mobility of the analyte. Therefore, individual components of a mixture will concentrate at different axial locations in the microchannel as long as they have different EP mobilities. To demonstrate this concept, we chose to separate a mixture of three fluorescent dyes: BODIPY ${ }^{2-}$, 8-methoxypyrene-1,3,6-trisulfonic acid (MPTS ${ }^{3-}$ ), and 1,3,6,8pyrene tetrasulfonic acid $\left(\mathrm{PTS}^{4-}\right)$. These dyes were chosen because they are fluorescent, are similarly sized, and possess different EP mobilities.

To demonstrate simultaneous concentration enrichment and separation of analytes, the three dyes listed above were prepared as a homogeneous solution in $5 \mathrm{mM}$ Tris buffer and loaded into a PDMS/glass microchannel containing a Au BPE. ${ }^{29}$ When an electric field of $7.5 \mathrm{kVm}^{-1}$ is applied, the dyes begin to concentrate in the anodic segment of the channel as evidenced by localized increases in fluorescence intensity. As shown in Figure 4e, three separate and resolved fluorescent bands are observed $\sim 200 \mathrm{~s}$ after the start of the experiment. The sequence of enrichment zones is in accordance with the EP mobilities of the analytes: PTS $^{4-}>$ MPTS $^{3-}>$ BODIPY $^{2-}$ (closest to the electrode) and the proposed model of concentration described in the previous section. Figure $4 \mathrm{f}$ is a plot of EF vs. channel position that corresponds to Figure 4e. The EFs for BODIPY2-, MPTS $^{3-}$, and PTS ${ }^{4-}$ in this figure are 560, 225, and 245, respectively. Similar results to these were achieved for two component separations in carbonate buffer, although slightly different behavior was observed because of a difference in electric field profiles arising in the two different buffers. ${ }^{29}$

\section{SUMMARY, CONCLUSIONS, AND OUTLOOK}

The bipolar behavior of floating electrodes experiencing a lateral electric field within microfluidic channels can be exploited for the development of analytical techniques and other applications. Here, we presented the use of BPEs for detection, concentration enrichment, and separation of analytes in a microfluidic environment. The characteristic phenomena that underlie these applications include the gradient of the potential difference between the electrode and the solution, the coupling of electrochemical reactions to ion flow, and modulation of the electric field inside the channel. 
a

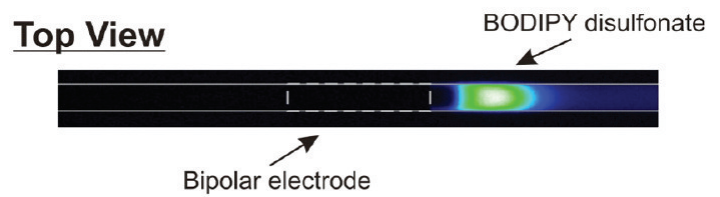

Side View

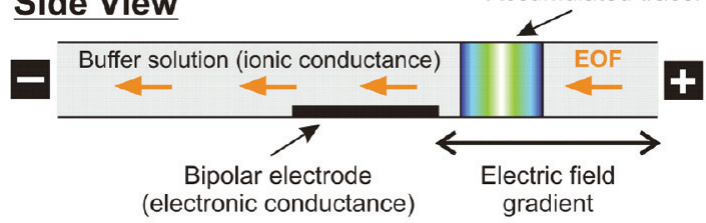

b

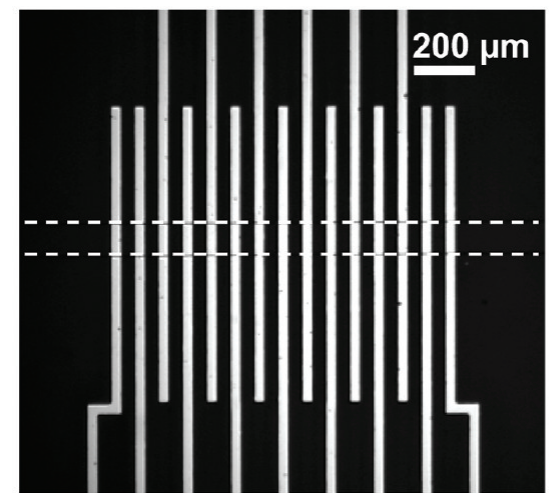

C
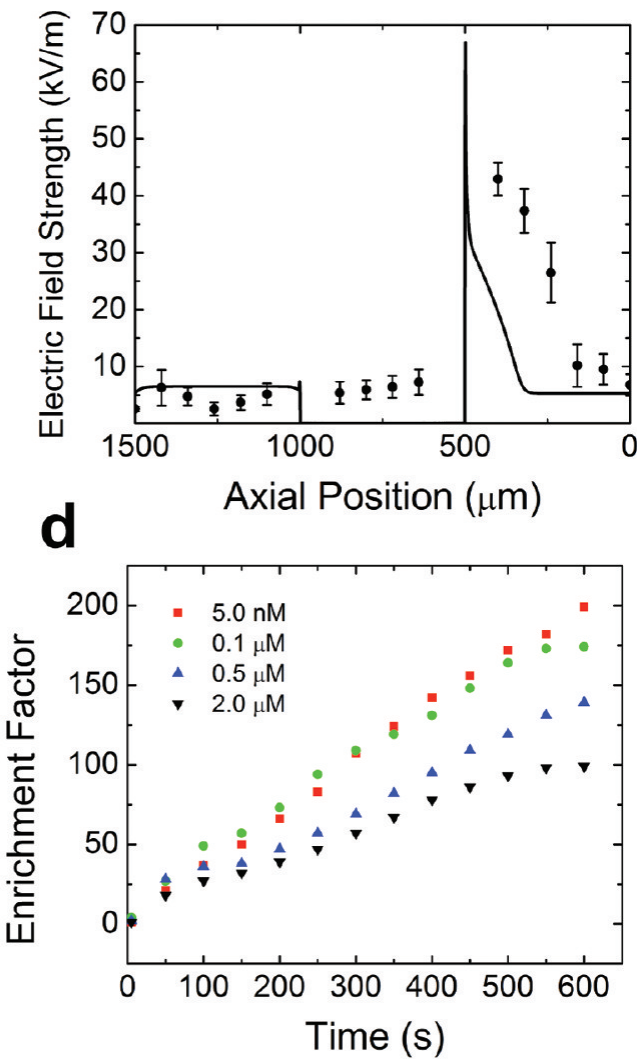

e BODIPY2-

MPTS ${ }^{3-}$ PTS ${ }^{4-}$

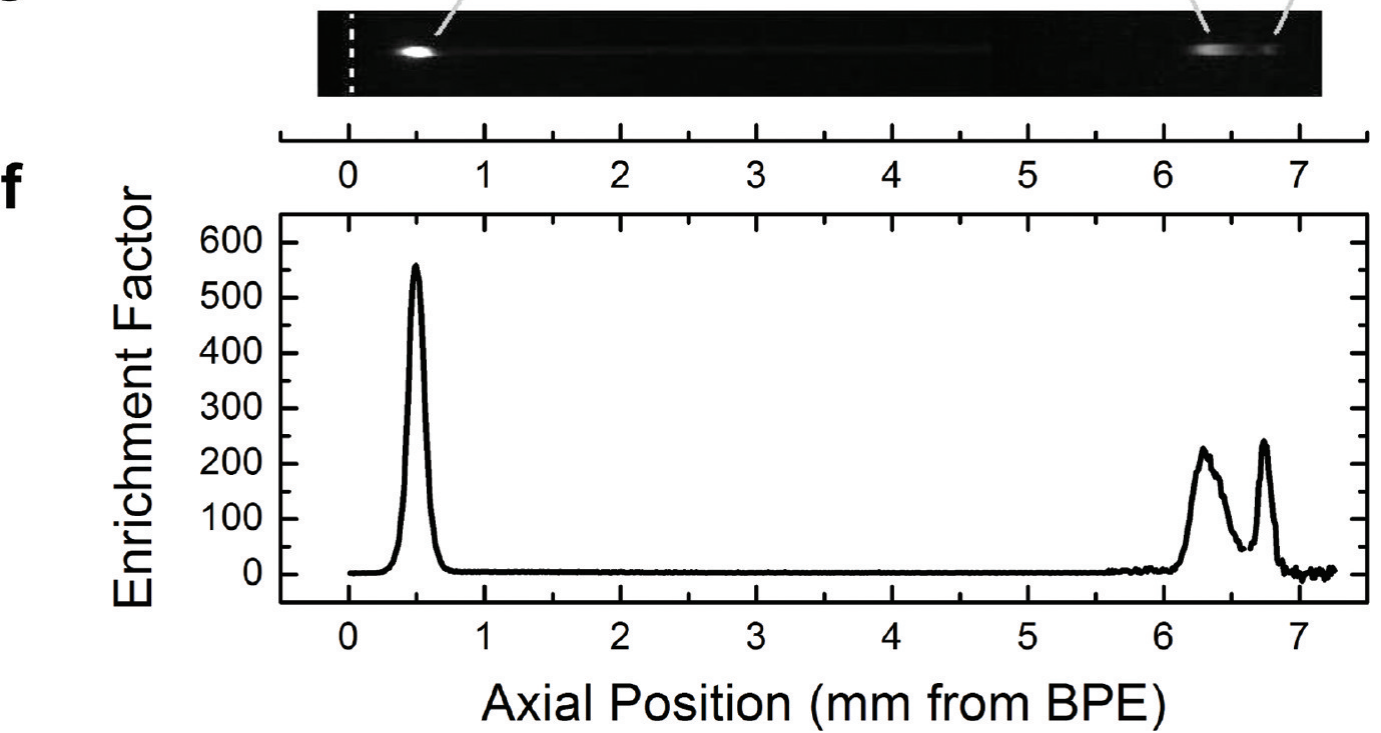

Figure 4. (a) Fluorescence micrograph (top view) and schematic diagram (side view) illustrating concentration enrichment of the fluorescent tracer BODIPY ${ }^{2-}$ to the right of a $500 \mu \mathrm{m}$-long Au BPE. (b) Optical micrograph of an array of 15 Au microelectrodes (40 $\mu \mathrm{m}$ lines and spaces) used to map the local electric field. The dashed white line indicates the location of the microchannel. (c) Local axial electric field strength obtained by numerical simulation (solid line) and experimental measurement (circles) using the device shown in part (b). The 2D simulation depicts the central $1.5 \mathrm{~mm}$ of a $20 \mu \mathrm{m}$-tall microchannel with the $0.5 \mathrm{~mm}$ BPE $(3.0 \mu \mathrm{m}$ tall) on the channel floor and extending from $\mathrm{x}=0$ to $-0.5 \mathrm{~mm}$. The initial condition is a uniform distribution of $5.0 \mathrm{mM}$ Tris buffer $(\mathrm{pH} 8.0)$. The zeta potential of the channel walls is assumed to be $-85 \mathrm{mV}$. Profiles represent the distribution of field strength along the channel at $\mathrm{y}=3.5 \mu \mathrm{m}$ and $\mathrm{t}=60 \mathrm{~s}$ after applying an average field strength of $5.83 \mathrm{kV} \mathrm{m}^{-1}$. An excess $8 \mu \mathrm{L}$ of the buffer in the cathodic reservoir stabilized the electric field during measurements. The data are averaged over two separate devices. In each case, several maps of the electric field were obtained using a different pair of microbands to define the BPE during each set of measurements. (d) EFs achieved for the BODIPY ${ }^{2-}$ tracer in $5 \mathrm{mM}$ Tris ( $\mathrm{pH}$ 8.0) with initial tracer concentrations of $5 \mathrm{nM}$ and $0.1,0.5$, and $2.0 \mu \mathrm{M}$. Applied field strength: $5.83 \mathrm{kV} / \mathrm{m}$. (e) Fluorescence micrograph (top view) showing separation of BODIPY ${ }^{2-}$, MPTS ${ }^{3-}$, and PTS $^{4-}$ in $5.0 \mathrm{mM}$ Tris Buffer $200 \mathrm{~s}$ after application of $E_{\text {tot }}=40 \mathrm{~V}$ (Omega Optical XF115-2 and XF02-2 filter sets overlayed) in a $12 \mathrm{~mm}-$ long, Pluronic-modified channel. (f) Plot of EF vs. axial location corresponding to the micrograph in (e). 
When the buffer concentration in the microchannel is high, most of the current moves through the solution and only $\sim 0.1 \%$ passes through the BPE. Under these conditions, the electric field is approximately linear throughout the channel, and therefore, it is possible to simultaneously control the potential of at least 1000 BPEs. Because the rates of the faradaic processes at the anodic and cathodic poles of a BPE must be equal, we showed that the current flowing through the BPE can be related to ECL intensity. This makes it possible to simultaneously read out the state of each electrode. A simpler and more sensitive detection method involves electrodissolution of $\mathrm{Ag}$. The key points are that the potential of an arbitrarily large number of electrodes can be controlled using this general method and the current flowing through each BPE can be determined simultaneously.

When the electrolyte concentration in the microchannel is low $(1-5 \mathrm{mM})$, more of the current is shunted through the BPE. This results in a local electric field gradient within the channel, and this can, in turn, lead to concentration enrichment. Analytes concentrate where their EP velocity exactly opposes their electroosmotic velocity. The former is a function of location-because of an extended field gradient-but because fluids are incompressible, the electroosmotic velocity is independent of local inhomogeneities in the field and only depends on the total applied voltage $\left(E_{t o t}\right)$. Therefore, mixtures of analytes having different EP mobilities concentrate at different locations along the field gradient, and this method can be used to both concentrate and separate analytes.

This method is incredibly simple: it requires a battery or simple power supply, microfabricated electrodes, and an appropriate electrochemical cell that can even be just a petri dish (Figure $2 b$ ). Because of this simplicity, BPEs likely could be profitably integrated into-and provide interesting functionality to - a broad range of lab-on-a-chip applications. Indeed, we have already shown that BPEs can be used to construct optoelectrochemical logic gates $^{36}$ and to obtain voltammograms without the need for a potentiostat. ${ }^{37}$ Although not discussed here, we have also shown that BPEs can be placed at the intersection of two fluidic channels and that this enables a whole new range of interesting functions. ${ }^{38}$ Perhaps the most exciting potential application of BPEs is integration into nanofluidic systems. The advantage is that they can be controlled without the need for a direct electrical connection, which is often a major obstacle for nanosystems.

\section{ACKNOWLEDGMENT}

We gratefully acknowledge financial support from the Chemical Sciences, Geosciences, and Biosciences Division, Office of Basic Energy Sciences, Office of Science, U.S. Department of Energy (Contract No. DE-FG02-06ER15758). We also acknowledge support from the U.S. Army Research Office (grant no. W911NF-071-0330) and the U.S. Defense Threat Reduction Agency. The Robert A. Welch Foundation (Grant F-0032) provides sustained support for our research. We also acknowledge major contributions to our studies of BPEs by our long-time collaborators Prof. Ulrich Tallarek and Dr. Dzmitry Hlushkou (Philipps-Universität Marburg). Finally, we thank three former colleagues who initiated our studies of BPEs a decade ago: Prof. Julio Alvarez (Virginia Commonwealth University), Dr. Li Sun (Pine Instruments), and Prof. Wei Zhan (Auburn University).
Richard M. Crooks is a professor of chemistry and holder of the Robert A. Welch Chair in Materials Chemistry at The University of Texas at Austin. Research in the Crooks lab focuses on electrochemistry, analytical microsystems, nanomaterials, and catalysis. Dr. François Mavré was a post-doctoral associate in the Crooks group for two years and now is an assistant professor of chemistry at Paris Diderot University. His research within the Laboratoire d'Electrochimie Moléculaire is focused on the development of electroanalytical strategies, including bioelectrochemical enzyme sensors, electrochemical real-time PCR, and aptasensors, for the detection of biomolecules. Robbyn K. Anand is a graduate student in the Crooks research group, but she will soon be moving to a posdoctoral position at the University of Washington. Her research interests include investigation of electrokinetic transport and electrochemistry in microfluidic devices. Dr. Derek Laws was a postdoctoral fellow in the Crooks lab for two years, and he is currently employed as an R\&D scientist at Siemens Water Technologies. His research interests include electroanalytical chemistry, bipolar electrochemistry, and electrochemistry of organometallic species. Dr. Kwok-Fan Chow received his Ph.D. from The University of Texas at Austin under the guidance of Prof. Richard M. Crooks. He is currently a postdoctoral research associate in Prof. Royce W. Murray's group at the University of North Carolina Chapel Hill. His research interests include analytical/physical electrochemistry and electrochemical properties of nanoparticles. Dr. Byoung-Yong Chang was a postdoctoral associate in the Crooks group for two years, and he just began his independent career as an assistant professor of chemistry at Pukyong National University in South Korea. His research interests are in the field of electrochemistry. John A. Crooks is currently working as a researcher in Dr. Amy Tang's lab at Eastern Virginia Medical School. His work focuses on cancer signaling pathways and locating novel therapeutic targets within the cell. Address correspondence to Crooks at Department of Chemistry and Biochemistry and the Center for Electrochemistry, The University of Texas at Austin, 1 University Station, A5300, Austin, Texas 78712-0165. Email: crooks@cm.utexas.edu.Telephone: 512-475-8674.

\section{REFERENCES}

(1) Bard, A. J.; Faulkner, L. R. Electrochemical Methods: Fundamentals and Applications, 2nd ed.; John Wiley \& Sons, Inc.: New York, 2001.

(2) Mavré, F.; Chow, K.-F.; Sheridan, E.; Chang, B.-Y.; Crooks, J. A.; Crooks, R. M. Anal. Chem. 2009, 81, 6218-6225.

(3) Duval, J. F. L.; Minor, M.; Cecilia, J.; van Leeuwen, H. P. J. Phys. Chem. B 2003, 107, 4143-4155.

(4) Duval, J.; Kleijn, J. M.; van Leeuwen, H. P. J. Electroanal. Chem. 2001, $505,1-11$.

(5) Fleischmann, M.; Ghoroghchian, J.; Rolison, D.; Pons, S. J. Phys. Chem. 1986, 90, 6392-6400.

(6) Ghoroghchian, J.; Pons, S.; Fleischmann, M. J. Electroanal. Chem. 1991, 317, 101-108.

(7) Smotkin, E.; Bard, A. J.; Campion, A.; Fox, M. A.; Mallouk, T.; Webber, S. E.; White, J. M. J. Phys. Chem. 1986, 90, 4604-4607.

(8) Smotkin, E. S.; Cerveramarch, S.; Bard, A. J.; Campion, A.; Fox, M. A.; Mallouk, T.; Webber, S. E. J. Phys. Chem. 1987, 91, 6-8.

(9) Cerveramarch, S.; Smotkin, E. S.; Bard, A. J.; Campion, A.; Fox, M. A.; Mallouk, T.; Webber, S. E.; White, J. M. J. Electrochem. Soc. 1988, 135, 567-573.

(10) Bradley, J.-C.; Chen, H.-M.; Crawford, J.; Eckert, J.; Ernazarova, K.; Kurzeja, T.; Lin, M.; McGee, M.; Nadler, W.; Stephens, S. G. Nature 1997, 389, 268-271.

(11) Wang, Y.; Hernandez, R. M.; Bartlett, D. J.; Bingham, J. M.; Kline, T. R.; Sen, A.; Mallouk, T. E. Langmuir 2006, 22, 10,451-10,456.

(12) Ulrich, C.; Andersson, O.; Nyholm, L.; Björefors, F. Angew. Chem.-Int. Edit. 2008, 47, 3034-3036.

(13) Ulrich, C.; Andersson, O.; Nyholm, L.; Björefors, F. Anal. Chem. 2009, $81,453-459$.

(14) Warakulwit, C.; Nguyen, T.; Majimel, J.; Delville, M. H.; Lapeyre, V.; Garrigue, P.; Ravaine, V.; Limtrakul, J.; Kuhn, A. Nano Lett. 2008, 8, 500504.

(15) Ramakrishnan, S.; Shannon, C. Langmuir , 26, 4602-4606.

(16) Nyholm, L. Analyst 2005, 130, 599-605.

(17) Klett, O.; Nyholm, L. Anal. Chem. 2003, 75, 1245-1250.

(18) Ordeig, O.; Godino, N.; del Campo, J.; Munoz, F. X.; Nikolajeff, F.; Nyholm, L. Anal. Chem. 2008, 80, 3622-3632.

(19) Arora, A.; Eijkel, J. C. T.; Morf, W. E.; Manz, A. Anal. Chem. 2001, 73, 3282-3288.

(20) Zhan, W.; Alvarez, J.; Crooks, R. M. J. Am. Chem. Soc. 2002, 124, 13,26513,270 .

(21) Miao, W. J. Chem. Rev. 2008, 108, 2506-2553.

(22) Zhan, W.; Alvarez, J.; Crooks, R. M. Anal. Chem. 2003, 75, 313-318. 
(23) Zhan, W.; Alvarez, J.; Sun, L.; Crooks, R. M. Anal. Chem. 2003, 75, 12331238.

(24) Chow, K.-F.; Mavré, F.; Crooks, R. M. J. Am. Chem. Soc. 2008, 130, 75447545.

(25) Chow, K.-F.; Mavré, F.; Crooks, J. A.; Chang, B.-Y.; Crooks, R. M. J. Am. Chem. Soc. 2009, 131, 8364-8365.

(26) Chow, K.-F.; Chang, B.-Y.; Zaccheo, B.; Mavré, F.; Crooks, R. M. J. Am. Chem. Soc. 2010, 132, 9228-9229.

(27) Dhopeshwarkar, R.; Hlushkou, D.; Nguyen, M.; Tallarek, U.; Crooks, R. M. J. Am. Chem. Soc. 2008, 130, 10,480-10,481.

(28) Hlushkou, D.; Perdue, R. K.; Dhopeshwarkar, R.; Crooks, R. M.; Tallarek, U. Lab on aChip 2009, 9, 1903-1913.

(29) Laws, D. R.; Hlushkou, D.; Perdue, R. K.; Tallarek, U.; Crooks, R. M. Anal. Chem. 2009, 81, 8923-8929.
(30) Perdue, R. K.; Laws, D. R.; Hlushkou, D.; Tallarek, U.; Crooks, R. M. Anal. Chem. 2009, 81, 10,149-10,155.

(31) Gebauer, P.; Bocek, P. Electrophoresis 2000, 21, 3898-3904.

(32) Jung, B.; Bharadwaj, R.; Santiago, J. G. Electrophoresis 2003, 24, 34763483.

(33) Ring-Ling, C. Electrophoresis 2003, 24, 486-497.

(34) Cheng-Huang, L.; Takashi, K. Electrophoresis 2004, 25, 4058-4073.

(35) Wei, W.; Xue, G.; Yeung, E. S. Anal. Chem. 2002, 74, 934-940.

(36) Zhan, W.; Crooks, R. M. J. Am. Chem. Soc. 2003, 125, 9934-9935.

(37) Chang, B.-Y.; Mavré, F.; Chow, K.-F.; Crooks, J. A.; Crooks, R. M. Anal. Chem. 2010, 82, 5317-5322.

(38) Fosdick, S. E.; Crooks, J. A.; Chang, B.-Y.; Crooks, R. M. J. Am. Chem. Soc. 2010, 132, 9226-9227.

AC101262V 